\title{
Assessing Evidence of Validity for the Intuitive Eating Scale-2 among Adult Latina Women
}

\author{
Hala Madanat ${ }^{1,2}$, Jessica Hawks ${ }^{3}$, Amanda Gonzales ${ }^{4}$, Eliana Miranda1, \\ Eric R. Walsh-Buhi' ${ }^{5}$ Michelle Takemoto6, Elinor Gaida ${ }^{2}$ \\ ${ }^{1}$ School of Public Health, San Diego State University, Campanile Dr., MC, San Diego, USA \\ ${ }^{2}$ Institute for Behavioral and Community Health, San Diego State University Research Foundation, San Diego, USA \\ ${ }^{3}$ San Diego State University/University of California San Diego Joint Doctoral Program in Public Health, San Diego, USA \\ ${ }^{4}$ Clinical and Translational Science Institute, University of California-Los Angeles, Los Angeles, USA \\ ${ }^{5}$ Indiana University School of Public Health-Bloomington, Bloomington, IN, USA \\ ${ }^{6}$ Department of Family Medicine and Public Health, University of California, San Diego, Gilman Drive, La Jolla, USA \\ Email: ^hmadanat@sdsu.edu
}

How to cite this paper: Madanat, H., Hawks, J., Gonzales, A., Miranda, E., Walsh-Buhi, E.R., Takemoto, M. and Gaida, E. (2020) Assessing Evidence of Validity for the Intuitive Eating Scale-2 among Adult Latina Women. Health, 12, 293-304.

https://doi.org/10.4236/health.2020.124024

Received: February 24, 2020

Accepted: March 28, 2020

Published: April 2, 2020

Copyright (c) 2020 by author(s) and Scientific Research Publishing Inc. This work is licensed under the Creative Commons Attribution International License (CC BY 4.0)

http://creativecommons.org/licenses/by/4.0/

\begin{abstract}
Introduction/Background: No research has been conducted on the relevance of intuitive eating as a promising strategy for sustainable weight management among Latinas. This study translated Tylka and Kroon Van Diest's (2013) Intuitive Eating Scale-2 (IES-2) into Spanish and employed an exploratory factor analysis (EFA) to examine evidence of validity. Methods: We recruited Latinas from community venues in South San Diego, California to participate in this cross-sectional study. Inclusion criteria were: at least 18 years of age, monolingual Spanish or bilingual English/Spanish speaker, and self-identified Latina. In total, 150 Latinas completed a structured, face-to-face interview, which included the Spanish IES-2. Data were used in conducting an EFA. Results: Four items were removed for not loading on the initial EFA. Once items were removed, we extracted five factors with Eigenvalues greater than 1, which accounted for almost two-thirds of the variance. Of the four original IES-2 factors, two were retained identically: $R e-$ liance on Hunger and Satiety Cues (RHSC) and Body-Food Choice Congruence (B-FCC). The Eating for Physical Rather than Emotional Reasons (EPR) subscale lost one item in the initial EFA, and was not unidimensional, resulting in two separate subscales. Evidence of score reliability was marginal to strong ( $\alpha=0.63-0.87)$. Discussion: This study is first to provide evidence of validity and reliability for a Spanish translation of the IES-2. The results suggest that the proposed Spanish IES-2 can be used to study intuitive eating behaviors and attitudes among Latinas. However, further research with larger sample sizes and other diverse populations are suggested.
\end{abstract}




\section{Keywords}

Intuitive Eating, Exploratory Factor Analysis, Latina, Dieting

\section{Introduction}

Overweight, obesity, and cardiovascular disease have a major impact on women of Hispanic or Latin American origins in the U.S. A disproportionate percentage of adult Latinas ${ }^{* 1}(77.2 \%)$ are overweight or obese, compared with $65.8 \%$ of non-Latina adult women [1]. The prevalence of obesity among Latinas is $44.4 \%$, whereas the overall obesity prevalence among women in the U.S. is much lower at $36.1 \%$ [1]. Latinas have the second highest prevalence of obesity of all ethnic groups behind non-Latina Black women [1]. Among specific priority populations, women of Mexican descent also have higher cholesterol levels (48.1\% have cholesterol higher than $200 \mathrm{mg} / \mathrm{dL}$ ) compared to non-Latina Whites (45.8\%) [2]. Overweight and obesity are related to the development of morbidity and mortality from cardiovascular disease, type II diabetes, and cerebrovascular disease [3].

Evidence suggests that changes in dietary behavior due to acculturation-the process by which individuals adapt to and adopt the dominant culture's beliefs, attitudes, and practices-may be partially responsible for the disproportionate prevalence of overweight and obesity among Latinas [4]. For example, more acculturated Puerto Ricans eat higher amounts of saturated fat, consume less starchy vegetable, legume, and cereal fiber than their less acculturated counterparts [5]. In their systematic review of acculturation and diet among Latinos, Ayala, Baquero, and Klinger [6] showed evidence that more acculturated individuals were more likely to eat added fats, fatty snacks, and fast food than those who were less acculturated. On the reverse, less acculturated Latinos tended to eat more fruit, rice, beans, and vegetables than Latinos who were more acculturated [6]. Findings indicate that Latinos' diet quality generally decreases as individuals become more acculturated, with the exception of higher vegetable intake [6].

Reasons for the change in diet quality may be explained by the actual and/or perceived differences in access to healthy foods in the U.S. compared to other Latin American countries. Qualitative metasynthesis examined Latinas' changes in diet since migrating to the U.S. [7]. The findings indicated that Latinas perceived a greater availability of junk food and fast food in the U.S. compared to their home countries. In addition, Latinas identified barriers to accessing healthy foods in the U.S. including high prices, not enough time to eat a healthy diet, and an over-abundance of processed food in the U.S. leading to weight gain [7]. Other research suggests food marketing directed towards Latinos is more likely to promote foods and drinks with added sugars and fast food than food marketing directed at non-Latinos [8].

${ }^{1}$ For consistency in this manuscript, we refer to all people of Spanish-speaking origin as Latina/as. 
In addition to the abundant availability and visibility of unhealthy food, Latinas are also exposed to media that promotes the "thinness ideal" (i.e., the preference for thinness as the ideal standard of beauty) [9]. The thinness ideal and anti-fat attitudes have been found to impact body satisfaction resulting in unhealthy dieting and weight loss attempts that can result in increased BMI and eating disorders [10]. As in the overall population, Latina's exposure to the thinness ideal in media can increase negative affect (such as anger and depression) while increasing body image dissatisfaction and emotional distress [9]. Furthermore, in Latinas, exposure to the thinness ideal can lead to conflicting messages: women should lose weight (message from mainstream U.S. culture, including health care professionals) and being overweight is ideal (message from Latina home culture) [11]. Pepper and Ruiz's [12] study of anti-fat attitudes, body image, and eating behaviors among Latinas indicated that Latinas with high acculturation were more likely than bicultural or low acculturation Latinas to report anti-fat attitudes. When examining the relationship between weight loss strategies and acculturation among Latinas, Marquez, Ayala, and Wing [13] found that reducing caloric intake to lose weight was correlated with more English spoken at home and length of time living in the U.S. was associated with a higher likelihood of increasing physical activity or reducing portion sizes to lose weight [13]. Additionally, greater acculturation was found to be associated with fat avoidance [6], weight dissatisfaction [14], and attempted weight loss [14].

The findings from Diaz et al. [11], Marquez et al. [13], New et al. [14], and Pepper \& Ruiz [12] suggest a need for a culturally appropriate and sustainable weight management strategy. Intuitive eating, a weight management strategy first developed by dieticians Tribole and Resch [15], represents a promising strategy. Intuitive eating is guided by a model of body acceptance and eating according to internal physical hunger cues rather than environmental, social, or emotional cues. Dieting, food restrictions and an ideal body image are removed. Some evidence suggests that intuitive eating is associated with decreased body mass index (BMI), improved psychological health (e.g., better body image, decreased levels of depression among women), improved LDL and HDL cholesterol, and potentially improved overall dietary quality (e.g., increased vegetable consumption and decreased binge eating) [16]. While other weight loss strategies based on restricting calories and/or certain food groups have demonstrated limited sustainability and efficacy for weight management [17], intuitive eating's non-restrictive approach is likely more sustainable for weight management [16] and more appropriate for a Latina audience with their unique cultural values and needs.

Intuitive eating has been studied among several different demographic groups, including college males and females [18] [19], international populations in Asia, Europe, and the Middle East [20] [21] [22] [23] [24], and adolescents [25] [26] [24]. However, to our knowledge, no research has been conducted on the relevance of intuitive eating to Latino populations in the U.S. 
To assess the level of intuitive eating among individuals, Tylka and Kroon Van Diest [27] developed the Intuitive Eating Scale-2 (IES-2). The IES-2 has 23 items with 4 separate constructs (i.e., eating for physical rather than emotional reasons (ERP), unconditional permission to eat (UPE), reliance on hunger and satiety cues (RHSC), and body-food choice congruence (B-FCC)) with factor loadings of at least 0.5 [27]. The scale has produced estimates of high internal consistency with a Cronbach's alpha of 0.88 and 0.89 for women and men, respectively, presenting improvements over the original IES-1 scale. Data collected with IES-2 have demonstrated sufficient evidence of construct validity, and IES-2 scores were negatively associated with eating disorder symptoms and body shame while positively associated with body appreciation [27]. The scale was developed to provide a tool for empirical research and clinical work in assessing and addressing weight management and eating disorders [27].

The objective of this study was to investigate whether a proposed Spanish translation of the IES- 2 can be used to study intuitive eating behaviors and attitudes among Latinas. This paper presents a Spanish IES-2 scale and reports on an exploratory factor analysis (EFA) to evaluate the scale's validity and reliability among Latina audiences in the U.S. We identify latent factors within this scale and provide empirical evidence for the inclusion of items in this adapted Spanish language version among adult Latinas. This research represents the first study to examine evidence of validity and reliability of a Spanish translation of the IES-2 (Spanish IES-2).

\section{Methods}

Design: We employed a single group cross-sectional study design.

Participants and Setting: The setting for this study was South San Diego, California including the communities of San Ysidro and Chula Vista. We approached Latinas in schools, community centers, and via existing social networks of Latinas who had participated in previous research projects, including ongoing free exercise classes delivered in the community. To be eligible, women had to be 18 years of age or older, either a monolingual Spanish speaker or bilingual English/Spanish speaker, and self-identify as Latina. We recruited 150 Latinas who completed a structured, face-to-face survey.

Instruments: The paper-and-pencil survey instrument consisted of the Intuitive Eating Scale-2 (IES-2) and demographic variables (age, country of birth, marital status, employment status, monthly family income, family size, and self-reported general health status). Following guidelines by Fowler [28], the IES-2 was translated to Spanish by a bilingual/bicultural certified translator, and independently back-translated to English by another bilingual/bicultural individual. Differences were reconciled and a final version (the Spanish IES-2) was reviewed by another bilingual/bicultural individual familiar with the construct of intuitive eating.

Data Collection: Face-to-face interviews were conducted by three trained bilingual/bicultural research assistants (RAs). RAs approached potential partici- 
pants and provided them with information on the study. If the individual was interested, she was then screened for eligibility. To obtain informed consent, the purpose of the study was explained to the women including that participation was voluntary, their names would not be requested, and their information would remain anonymous. The survey was self-administered, i.e. each interviewee recorded her answers on the instrument directly. Prior to the start of the study, the Institutional Review Board at the lead authors' institution reviewed and approved the study.

Data Analysis: Data were analyzed using IBM ${ }^{\circ}$ SPSS Statistics (Release 21.0.0.0). Descriptive statistics and exploratory factor analyses (EFAs) were used to analyze the data. Due to the structured, face-to-face interview format, there were no missing data.

Preliminary analyses included an examination of inter-item correlations and evidence of normality. EFA was conducted by using maximum likelihood analysis. After removing all items that had item-to-factor correlations lower than 0.40 based on Raubenheimer's [29] item selection procedure, we determined the number of retained factors based on a factor having an Eigenvalue greater than 1 [30]. We employed varimax orthogonal rotation [30] and assessed inter-factor correlations. Internal consistency of the Spanish IES-2 subscales was assessed with the Cronbach's alpha. Values between 0.6 and 0.7 are considered marginal, those above 0.7 are considered acceptable, with value of 0.8 and 0.9 being preferred [31]. We also assessed inter-item correlations within each scale. For the scale to be considered unidimensional, the mean interim correlations fall between 0.15 and 0.50 [32].

\section{Results}

Table 1 presents the participants' demographic characteristics. The mean age of the 150 participants was 49.15 years $(S D=13.98)$. The majority were married or living with a partner (56.7\%), born in Mexico (77.3\%), homemakers (52.7\%), with a monthly family income of less than $\$ 2000$ (58.7\%), and reported at least good overall health (60.3\%).

Preliminary analyses (i.e., testing of assumptions for factor analysis) determined the data were suitable for the EFA. The sample size exceeded the 5:1 case to item minimum requirement [33]. All variable data were approximately normally distributed. We removed four items for not loading on the initial EFA. These items were (item 1, I try to avoid certain foods high in fat, carbohydrates or calories, item 4, I get mad at myself for eating something unhealthy, item 9, I have forbidden foods that I don't allow myself to eat, and item 15, I find other ways to cope with stress and anxiety rather than by eating). Once items were removed, we extracted five factors with Eigenvalues greater than 1, which accounted for almost two-thirds of the total variance explained $(25.8 \%, 13.7 \%$, $11.2 \%, 6.9 \%$, and $6.6 \%)$. Since each of the five factors had a variance greater than $5 \%$, all were retained. The Cronbach's alpha coefficient for the five scales ranged 
from 0.63 to 0.87 . Only one factor had a Cronbach's alpha coefficient of less than 0.70 , which is considered marginal. All other factors had Cronbach alpha coefficients above 0.75 , indicating strong internal consistency.

Table 1. Distribution of participants according to select demographic variables $(\mathrm{N}=150)$.

\begin{tabular}{|c|c|}
\hline Variable & n (Percentage \\
\hline \multicolumn{2}{|l|}{ Age } \\
\hline \multicolumn{2}{|l|}{ Marital Status } \\
\hline Married/lives with Partner & $85(56.7 \%)$ \\
\hline Single & $19(12.7 \%)$ \\
\hline Widowed & $17(11.3 \%)$ \\
\hline Divorced & $14(9.3 \%)$ \\
\hline Separated & $15(10.0 \%)$ \\
\hline \multicolumn{2}{|l|}{ Country of birth* } \\
\hline United States & $29(19.3 \%)$ \\
\hline Mexico & $116(77.3 \%)$ \\
\hline Other & $4(2.7 \%)$ \\
\hline \multicolumn{2}{|l|}{ Employment Status } \\
\hline Employed & $50(33.3 \%)$ \\
\hline Home-maker & $79(52.7 \%)$ \\
\hline Retired & $12(8.0 \%)$ \\
\hline Not currently working & $9(6.0 \%)$ \\
\hline \multicolumn{2}{|l|}{ Monthly Family Income } \\
\hline$<\$ 500$ & $18(12.0 \%)$ \\
\hline$\$ 500-999$ & $15(10.0 \%)$ \\
\hline$\$ 1000$ - 1499 & $28(18.7 \%)$ \\
\hline$\$ 1500-1999$ & $27(18.0 \%)$ \\
\hline$\$ 2000-2499$ & $11(7.3 \%)$ \\
\hline$\$ 2500-2999$ & $8(5.3 \%)$ \\
\hline$\$ 3000$ - 3499 & $8(5.3 \%)$ \\
\hline$\$ 3500-3999$ & $9(6.0 \%)$ \\
\hline$>\$ 4000$ & $13(8.6 \%)$ \\
\hline Don't know & $13(8.7 \%)$ \\
\hline
\end{tabular}

Number of individuals supported by income

\section{Self-rated Health}

$\begin{array}{cc}\text { Excellent } & 6.00 \% \\ \text { Very Good } & 15.30 \% \\ \text { Good } & 39.30 \% \\ \text { Regular } & 30.00 \% \\ \text { Poor } & 9.30 \%\end{array}$

${ }^{*}$ one person report not knowing her country of birth. 
Of the four IES-2 factors identified by Tylka and Van Diest [27], two factors were retained identically. These included the Reliance on Hunger and Satiety Cues (RHSC) subscale and Body-Food Choice Congruence (B-FCC) subscale. The Eating for Physical Rather than Emotional Reasons (EPR) subscale lost one item in the initial EFA (Item 15, I find other ways to cope with stress and anxiety than by eating), and was not unidimensional, resulting in two separate subscales one (ERP-1) with regular coded items (items 2, 5, 10, and 11 with wording of healthy eating behavior) and the second subscale (ERP-2) including only reverse-coded items (items 12,13, and 14 with wording of unhealthy eating behavior). Finally, the Unconditional Permission to Eat (UPE) subscale lost three of its six items and was the subscale with the lowest Cronbach alpha coefficient at 0.63. Factors, Cronbach alpha coefficients, and item loadings are presented in Table 2.

Table 2. Exploratory factor analysis: factor loadings and cronbach's alpha coefficients.

Factor. Reliance on Hunger and Satiety Cues (RHSC)

Alpha Loading

Item 6: Confío en que mi cuerpo me diga cuándo comer.

Item 7: Confio en que mi cuerpo me diga que comer.

Item 8: Confío en que mi cuerpo me diga cuánto comer.

Item 21:Confío en que mis señales de hambre me digan cuándo comer.

Item 22: Confío en que sentirme llena (mi saciedad) me diga cuándo dejar de comer.

Item 23: Confío en que mi cuerpo me diga cuándo dejar de comer.

\section{Factor. Body-Food Choice Congruence (B-FCC)}

Item 18: La mayoría del tiempo yo deseo de comer alimentos nutritivos.

Item 19: Generalmente como alimentos que hacen que mi cuerpo funcione eficientemente (bien).

Item 20: Generalmente como alimentos que le dan energía y resistencia a mi cuerpo.

Item 2: Me encuentro comiendo cuando siento emociones (como ansiedad, depresión o tristeza), aun cuando físicamente no tengo hambre.

Item 5: Me encuentro comiendo cuando me siento sola, aun cuando físicamente no tengo hambre.

Item 10: Uso la comida para ayudarme a calmar mis emociones negativas.

Item 11: Me encuentro comiendo cuando estoy estresada, aun cuando físicamente no tengo hambre. 


\section{Discussion}

As the prevalence of overweight and obesity among Latinas in the U.S. increases, it is important to identify methods to address this situation. It is proposed that accurately and reliably measure intuitive eating behaviors and related attitudes and interventions to increase intuitive eating among Latinas may be an effective strategy to address overweight and obesity in Latinas. The exploratory factor analysis of the Spanish IES-2 revealed five latent factors: Reliance on Hunger and Satiety Cues (RHSC), Body-Food Choice Congruence (B-FCC), Eating for Physical Rather than Emotional Reasons (EPR-1 and EPR-2), and Unconditional Permission to Eat (UPE). Both the RHSC and B-FCC factors were retained identically from the original IES-2 [27]. Eating for Physical Rather than Emotional Reasons was separated into two latent factors, one reverse coded and one coded regularly. The Unconditional Permission to Eat scale lost three items from the original IES-2 and its internal consistency was reduced.

This and past validation research of the IES-2 shows the need to consider changes to latent factors and items for unique priority population tested. For example, when translated to French and tested among a nationally representative sample of adults in France, only 3 subscales and 18 of the original 23 IES-2 items were retained (Reliance on Hunger and Satiety Cues, Eating for Physical Rather than Emotional Reasons, and Unconditional Permission to Eat) [20]. When the IES- 2 was validated among a sample of U.S. adolescents, two subscales (Unconditional Permission to Eat and Eating for Physical Rather than Emotional Reasons) were retained identically [25]. Reliance on Hunger and Satiety Cues from the original IES-2 was split to form two subscales, Awareness of Internal Hunger/Satiety Cues and Trust in Internal Hunger/Satiety Cues [25]. In summary, the factor Eating for Physical Rather than Emotional Reasons was retained identically in the Spanish IES-2, the French IES-2, and the adolescent IES-2. Further research is needed to understand the differences seen when validating the original IES-2 in other populations, including Spanish-speaking Latinas. These results suggest the need for adjustment of the IES-2 scale, especially for factors UPE, RHSC, and B-FFC. It is likely that item and factor changes are needed for different audiences and culturally unique populations including Latinas and Latinos in the U.S.

The current findings indicate the IES-2 Spanish translation to be a useful tool to study intuitive eating among Latinas in the U.S. However, the lower validity of the Unconditional Permission to Eat (UPE) latent factor and the split of the Eating for Physical Rather than Emotional Reasons (ERP) into two factors, suggests further refinement and adaptation to create a scale with higher validity for Latina populations may be needed. Insights about Latinas' unique cultural values and behaviors related to diet should be used to adapt the psychometric characteristics of intuitive eating to develop a culturally and linguistically appropriate Intuitive Eating Scale for Latinas (IES-Latino). Acculturation among Latinas has been found to be associated with different eating behaviors and perceptions re- 
lated to body images [6] [14]. Additionally, food insecurity in low-income Latino households may impact eating behaviors including intuitive eating potentially resulting in needs for adapted scale items [27]. Thus, future research should also investigate differences in psychometric characteristics due to relevant population attributes such as level of acculturation, poverty (food scarcity), among others. The objective of this research would be to test and validate an adapted version of the current Spanish IES-2. Researchers should also consider using the English version of the IES-2 among English speaking Latinas.

This combined new body of knowledge could provide a solid understanding of eating characteristics among Latinas and deliver a toolkit to researchers and public health professionals to study and assess intuitive eating among Latina populations. This audience has a higher risk of being overweight and obese and suffers of associated increased morbidity and mortality from cardiovascular disease, type II diabetes, and cerebrovascular disease [3]. Identifying effective and sustainable weight management solutions is of imperative importance.

As with Latinas, Latino males are at greater risk of obesity [1] and resulting disease. Hence, further research into intuitive eating should also include Latino males. Both English and Spanish language scales should be explored for their applicability and validity with this audience. While findings by Tylka et al. [27] suggest that the IES- 2 can be used for white men and females, this may not be the case for Latinos and Latinas. Tylka et al. [27] noted moderate differences by sex for the Eating for Physical Rather than Emotional Reasons (ERP) factor and suggested this was due to different cultural body image and beauty expectations between women and men in the Western cultures. Different expectations and values for males and females also exist in the Latino culture and may affect validity of the scale. Furthermore, differences in acculturation for males and females as they adapt to Western values and lifestyle may impact the validity of a shared scale.

The development and validation of appropriate Intuitive Eating Scales such as the Spanish IES-2 are important steps in assessing intuitive eating among Latino audiences. A valid and reliable measuring tool is essential to allow researchers and public health professionals understand the association of intuitive eating with eating and body related measures such as BMI, body satisfaction and image, and eating disorders. This knowledge will be instrumental in understanding changes in eating behavior with increasing acculturation (longitudinal studies are needed) and the development of intuitive eating guidelines and programs to providing effective and sustainable weight management solutions to address obesity among Latinos.

The results of this factor analysis should be considered within the context of the limitations and strengths of this study. One limitation of this study may be its smaller sample size. However, our sample size met the minimum requirements for exploratory analysis and hence was deemed sufficient for the presented study. In addition, our sample was a convenience rather than a random 
sample, so the proposed modifications to the IES-2 may not be generalizable to Latina populations outside of the South San Diego County region. Further, our study recruited only women and, accordingly, is limited to only Latinas. We recommend assessment of the Spanish IES-2 among males. In accordance with the outlined future direction, further validation research with larger and more diverse sample populations should be conducted to firmly establish a Latino appropriate Intuitive Eating Scale for further empirical research and clinical work.

\section{Conclusion}

This study is the first to provide evidence of validity and reliability for a Spanish translation of the IES-2. The results of this study suggest that the proposed Spanish IES-2 can be used to study intuitive eating behaviors and attitudes among Latinas. However, further research is needed to understand why the Unconditional Permission to Eat subscale had lower internal consistency with some items not loading onto the subscale in the Latino population and whether scale adjustments to address cultural differences of this population would improve the validity of the scale. Further research should also examine the Spanish IES-2 in more diverse Latino populations including both sexes and other characteristics such as acculturation and food security. Finally, intuitive eating should be measured across time to understand how intuitive eating behaviors and attitudes change over time.

\section{Acknowledgements}

This research was supported by a grant from the Kaiser Family Foundation and by the National Cancer Institute of the National Institutes of Health under award numbers: U54CA132384 (SDSU) \& U54CA132379 (UC San Diego).

\section{Conflicts of Interest}

The authors declare no conflicts of interest regarding the publication of this paper.

\section{References}

[1] Ogden, C.L., Carroll, M.D., Kit, B.K. and Flegal, K.M. (2014) Prevalence of Childhood and Adult Obesity in the United States, 2011-2012. JAMA, 311, 806-814. https://doi.org/10.1001/jama.2014.732

[2] Daviglus, M.L., Pirzada, A. and Talavera, G.A. (2014) Cardiovascular Disease Risk Factors in the Hispanic/Latino Population: Lessons from the Hispanic Community Health Study/Study of Latinos (HCHS/SOL). Progress in Cardiovascular Diseases, 57, 230-236. https://doi.org/10.1016/j.pcad.2014.07.006

[3] Heron, M. (2013) Deaths: Leading Causes for 2010. National Vital Statistics Reports (Vol. 62) Hyattsville.

http://www.cdc.gov/nchs/fastats/lcod.htm $\backslash n$ http://www.ncbi.nlm.nih.gov/pubmed/24364902

[4] Berry, J. (2003) Conceptual Approaches to Acculturation. In: Chun, K., Organista, 
P.B. and Marin, G., Eds., Acculturation: Advances in Theory, Measurement, and Applied Research, American Psychological Association, Washington DC, 17-37.

[5] Van Rompay, M.I., McKeown, N.M., Castaneda-Sceppa, C., Falcón, L.M., Ordová, J.M. and Tucker, K.L. (2012) Acculturation and Sociocultural Influences on Dietary Intake and Health Status among Puertorican Adults in Massachusetts. Journal of the Academy of Nutrition and Dietetics, 112, 64-74.

https://doi.org/10.1016/j.jada.2011.08.049

[6] Ayala, G.X., Baquero, B. and Klinger, S. (2008) A Systematic Review of the Relationship between Acculturation and Diet among Latinos in the United States: Implications for Future Research. Journal of the American Dietetic Association, 108, 1330-1344. https://doi.org/10.1016/j.jada.2008.05.009

[7] Gerchow, L., Tagliaferro, B., Squires, A., Nicholson, J., Savarimuthu, S.M., Gutnick, D. and Jay, M. (2014) Latina Food Patterns in the United States: A Qualitative Metasynthesis. Nursing Research, 63, 182-193. https://doi.org/10.1097/NNR.0000000000000030

[8] Adeigbe, R.T., Baldwin, S., Gallion, K., Grier, S. and Ramirez, G. (2015) Food and Beverage Marketing to Latinos: A Systematic Literature Review. Health Education \& Behavior, 42, 569-568. https://doi.org/10.1097/NNR.0000000000000030

[9] Birkeland, R., Thompson, J.K., Herbozo, S., Roehrig, M., Cafri, G. and van den Berg, P. (2005) Media Exposure, Mood, and Body Image Dissatisfaction: An Experimental Test of Person versus Product Priming. Body Image, 2, 53-61. https://doi.org/10.1016/j.bodyim.2004.11.002

[10] Culbert, K., Racine, S. and Klump, K. (2015) Research Review: What We Have Learned about the Causes of Eating Disorders-A Synthesis of Sociocultural, Psychological, and Biological Research. Journal of Child Psychology and Psychiatry and Allied Disciplines, 56, 1141. https://doi.org/10.1111/jcpp.12441

[11] Diaz, V.A., Mainous, A.G. and Pope, C. (2007) Cultural Conflicts in the Weight Loss Experience of Overweight Latinos. International Journal of Obesity, 31, 328-333. https://doi.org/10.1038/sj.ijo.0803387

[12] Pepper, A.C. and Ruiz, S.Y. (2007) Acculturation's Influence on Antifat Attitudes, Body Image and Eating Behaviors. Eating Disorders, 15, 427-447.

https://doi.org/10.1080/10640260701667912

[13] Marquez, B., Ayala, G.X. and Wing, R.R. (2013) Acculturation and Weight Loss Strategies among Latinas. Journal of Immigrant and Minority Health, 17, 610-613. https://doi.org/10.1007/s10903-013-9936-Z

[14] New, C., Xiao, L. and Ma, J. (2013) Acculturation and Overweight-Related Attitudes and Behavior among Obese Hispanic Adults in the United States. Obesity, 21, 2396-2404. https://doi.org/10.1002/oby.20146

[15] Tribole, E. and Resch, E. (2012) Intuitive Eating: A Revoluationary Program That Works. 3rd Edition, St. Martin's Griffin, New York.

[16] Van Dyke, N. and Drinkwater, E.J. (2014) Relationships between Intuitive Eating and Health Indicators: Literature Review. Public Health Nutrition, 17, 1-10. https://doi.org/10.1017/S1368980013002139

[17] Mann, T., Tomiyama, A.J., Westling, E., Lew, A.M., Samuels, B. and Chatman, J. (2007) Medicare's Search for Effective Obesity Treatments: Diets Are Not the Answer. The American Psychologist, 62, 220-233. https://doi.org/10.1037/0003-066X.62.3.220

[18] Gast, J., Campbell, N.A., Hunt, A. and Leiker, J.J. (2015) Intuitive Eating: Associa- 
tions with Physical Activity Motivation and BMI. American Journal of Health Promotion, 9, e91-e99. https://doi.org/10.4278/ajhp.130305-QUAN-97

[19] Hawks, S., Madanat, H., Hawks, J. and Harris, A. (2005) The Relationship between Intuitive Eating and Health Indicators among College Women. American Journal of Health Education, 36, 331-336. https://doi.org/10.1080/19325037.2005.10608206

[20] Camilleri, G.M., Méjean, C., Bellisle, F., Andreeva, V.A., Sautron, V., Hercberg, S. and Péneau, S. (2015) Cross-Cultural Validity of the Intuitive Eating Scale-2. Psychometric Evaluation in a Sample of the General French Population. Appetite, 84, 34-42. https://doi.org/10.1016/j.appet.2014.09.009

[21] Hawks, S.R., Merrill, R.M., Madanat, H.N., Miyagawa, T., Suwanteerangkul, J., Guarin, C.M. and Shaofang, C. (2004) Intuitive Eating and the Nutrition Transition in Asia. Asia Pacific Journal of Clinical Nutrition, 13, 194-203.

[22] Madanat, H.N. and Hawks, S.R. (2004) Validation of the Arabic Version of the Intuitive Eating Scale. Promotion \& Education, 11, 152-157. https://doi.org/10.1177/175797590401100302

[23] Madden, C., Leong, S.L., Gray, A. and Horwath, C.C. (2012) Eating in Response to Hunger and Satiety Signals Is Related to BMI in a Nationwide Sample of 1601 Mid-Age New Zealand Women. Public Health Nutrition, 15, 2272-2279. https://doi.org/10.1017/S1368980012000882

[24] Wirtz, A. and Madanat, H. (2012-2013) Westernization, Intuitive Eating, and BMI: An Exploration of Jordanian Adolescents. International Quarterly of Community Health Education, 33, 275-287. https://doi.org/10.2190/IQ.33.3.d

[25] Dockendorff, S., Petrie, T., Greenleaf, C. and Martin, S. (2012) Intuitive Eating Scale: An Examination among Early Adolescents. Journal of Counseling Psychology, 59, 604-611. https://doi.org/10.1037/a0029962

[26] Healy, N., Joram, E., Matvienko, O., Woolf, S. and Knesting, K. (2015) Impact of an Intuitive Eating Education Program on High School Students' Eating Attitudes. Health Education, 115, 214-228. https://doi.org/10.1108/HE-03-2014-0043

[27] Tylka, T.L. and Kroon Van Diest, A.M. (2013) The Intuitive Eating Scale-2: Item Refinement and Psychometric Evaluation with College Women and Men. Journal of Counseling Psychology, 60, 137-153. https://doi.org/10.1037/a0030893

[28] Fowler, F.J. (2002) Survey Research Methods. 3rd Edition, Sage, Thousand Oaks.

[29] Raubenheimer, J. (2004) An Item Selection Procedure to Maximise Scale Reliability and Validity. SA Journal of Industrial Psychology, 30, 59-64. https://doi.org/10.4102/sajip.v30i4.168

[30] Thompson, B. (2004) Exploratory and Confirmatory Factor Analysis: Understanding Concepts and Applications. APA, Washington DC. https://doi.org/10.1037/10694-000

[31] DeVellis, R. (2003) Scale Development: Theory and Applications. 2nd Edition, Sage, Thousand Oaks.

[32] Clark, L.A. and Watson, D. (1995) Constructing Validity: Basic Issues in Objective Scale Development. Psychological Assessment, 7, 309-319. https://doi.org/10.1037/1040-3590.7.3.309

[33] Tabachnick, B.G. and Fidell, L.S. (2013) Using Multivariate Statistics. 6th Edition, Pearson, Boston. 